\title{
Determining the minor loss coefficient of cone diffusers
}

\author{
Gencho Popov ${ }^{1, *}$, Kliment Klimentov ${ }^{2}$, Boris Kostov, ${ }^{3}$ and Reneta Dimitrova ${ }^{4}$ \\ ${ }^{1}$ Prof., PhD, Department of Heat, Hydraulics and Environmental Engineering, University of Ruse, \\ Ruse 7017, Bulgaria \\ ${ }^{2}$ Assoc. Prof., PhD, Department of Heat, Hydraulics and Environmental Engineering, University of \\ Ruse, Ruse 7017, Bulgaria \\ ${ }^{3}$ Assist.. Prof., PhD, Department of Heat, Hydraulics and Environmental Engineering, University of \\ Ruse, Ruse 7017, Bulgaria \\ ${ }^{4} \mathrm{PhD}$ Stud, Department of Heat, Hydraulics and Environmental Engineering, University of Ruse, \\ Ruse 7017, Bulgaria
}

\begin{abstract}
This work represents results concerning different methods, used to determine the minor loss coefficient of a cone diffuser, installed at the system's outlet. These results are compared with experimental data and numerical results, obtained after using ANSYS CFX and the data base of Pipe Flow Expert. For determining the minor loss coefficient of a cone diffuser, being installed at the system outlet and having a straight pipe section in front of it, some models in ANSYS CFX are established. These models are validated by using previously published experimental data. An empirical equation, based on the results found, enabling the accomplishment of analytical studies concerning the energy efficiency of fan systems, using an outlet diffuser, has been established.
\end{abstract}

\section{Introduction}

According to [1], the fan system dynamic pressure represent from 10 to $30 \%$ of the total pressure, concerning the operating zone of the fan (pressure) curve. This requires for a part of the dynamic pressure to be converted into static pressure, which aims to decrease the (kinetic) energy loss at the fan system outlet. In practice this may be achieved by installing a diffuser at the fan outlet, in case that it is an aspiration system. Usually, in case of a combined or booster system, the fan has been installed at the system outlet. In some cases, there may be installed two diffusers - at the fan and system outlets.

It is well-known that, when providing same work conditions and using a diffuser, installed at the system outlet, the flow rate will be increased compared with a case of not using a diffuser. Sometimes it may turn out that the increased flow rate work regime (point 2 ' in fig. 1) is more energy efficient than the initial work regime - without using a diffuser (point 1 in fig.1). Furthermore, there is an option for achieving an additional energy saving when by decreasing the speed of rotation (from $n_{1}$ to $n_{2}-$ fig. 1 ) the diffuser outlet flow rate is restored to its initial value, but obtained at lower pressure - point 2 in fig. 1.

\footnotetext{
* Corresponding author: gspopov@uni-ruse.bg
} 


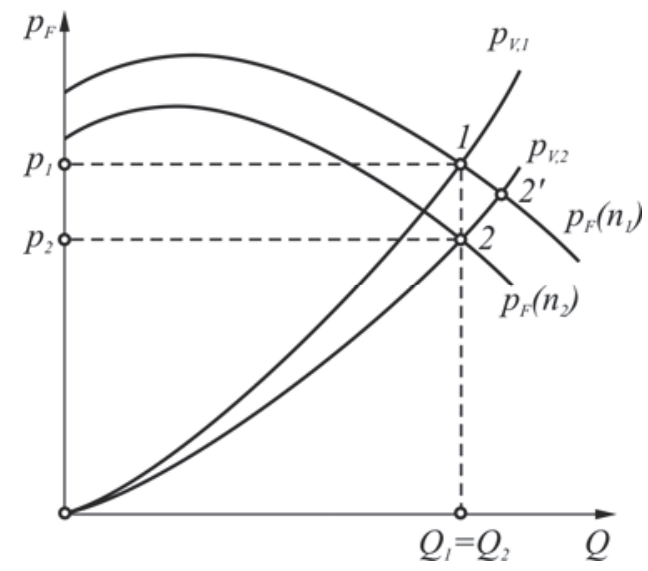

Fig. 1. Changing the operating (work) regime of a fan system: point $1-$ without using an outlet diffuser; point 2 - by using an outlet diffuser; point 2 - by using an outlet diffuser and restoring the initial system flow rate.

Assuming that all the existing system resistances are referred to the average velocity $v$, concerning the last straight pipe section, the pressure difference between $p_{1}$ and $p_{2}$ (fig,1) may be determined by using the following equation:

$$
p_{1}-p_{2}=\Delta p=\left(\alpha_{\text {out }}-\zeta_{d, \text { out }}\right) \rho \frac{v^{2}}{2},
$$

where $\alpha_{\text {out }}$ is the coefficient of the kinetic energy (Coriolis) at the system outlet, without using a diffuser; $\zeta_{d, \text { out }}$ - the minor loss coefficient of the system outlet diffuser; $\rho$ - the density of the transported fluid. It has to be indicated that the outlet diffuser minor loss coefficient includes the kinetic energy loss, obtained after the diffuser outlet [2].

Equation (1) may be presented into the following way:

$$
\frac{\Delta p}{p_{\text {din }}}=\alpha_{\text {out }}-\zeta_{d, \text { out }}
$$

where $p_{d i n}=\rho \frac{v^{2}}{2}$ is the fan dynamic pressure, in case that its inlet surface area is equal to the surface area of the last straight pipe section.

Equation (1) clearly indicates that using a diffuser for increasing the energy efficiency of a fan system is argued in case that the outlet diffuser minor loss coefficient $\zeta_{d, \text { out }}$ is lower than the coefficient of the kinetic energy (Coriolis) $\alpha_{\text {out }}$ at the system outlet, without using a diffuser, i.e. $\zeta_{d, \text { out }}<\alpha_{\text {out }}$.

For a more in-depth analytical study, which aim is to determine the impact of the diffuser on the fan systems energy efficiency, it is convenience to use analytical equations providing a connection between the diffuser geometric parameters and its minor loss coefficient $\zeta_{d, \text { out }}$. Such equations may be found in the existing literature sources, as well as experimental data published in tabular form. In this current work, it is accomplished a comparison between the results obtained by using several of the previously mentioned equations. Based on different types of diffusers, designed by using ANSYS CFX, it has been established some empirical equations valid for cases for which they are missing. 


\section{Equations used to determine the minor loss coefficient of cone diffusers}

It is well-known that, there are several parameters having impact on the energy loss, obtained in the diffuser [2, 3]: the diffuser degree of expansion (area ratio) $n=\frac{D^{2}}{d^{2}}$ (fig.2); the angle of expansion $\alpha$; the shape of the cross-section area; the Reynolds number $R e$ and the velocity profile at the diffuser inlet. In case that the diffuser is installed between two pipes, having different diameters, there are different equations that may be used for estimating the its minor loss coefficient $\zeta_{d}$. For example, it may be used the following equation [2]:

$$
\zeta_{d}=3.2\left(\operatorname{tg} \frac{\alpha}{2}\right) 1.25\left(1-\frac{1}{n}\right)^{2}+\frac{\lambda}{8 \sin \frac{\alpha}{2}}\left(1-\frac{1}{n^{2}}\right)
$$

where $\lambda$ is the major loss coefficient, which can be determined by using well-known hydraulic equations, as for this aim it is necessary to determine the fluid flow regime of motion, as well as the diffuser walls roughness.

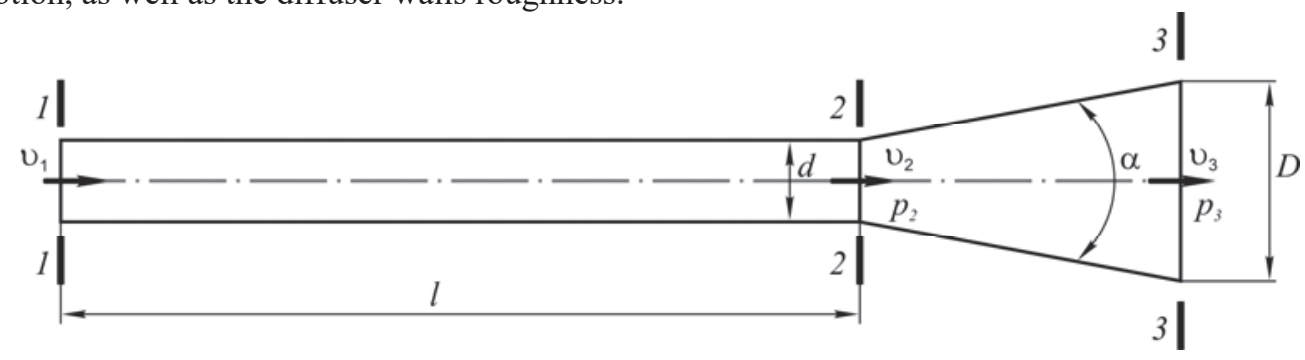

Fig. 2. Computational scheme of a system with an installed outlet diffuser

Another option the estimation of $\zeta_{d}$, given in $[2,3]$, is the following one:

$$
\zeta_{d}=\frac{\lambda}{8 \sin \frac{\alpha}{2}}\left(1-\frac{1}{n^{2}}\right)\left(1+\frac{0.5}{1.5^{\tilde{x}}}\right)+\Phi\left(1-\frac{1}{n}\right)^{1.92}
$$

where $\Phi=0.024 \alpha ; \quad \tilde{x}=\frac{\ln \left(1+2 \bar{L} \operatorname{tg} \frac{\alpha}{2}\right)}{2 \operatorname{tg} \frac{\alpha}{2}}$. The diffuser relative length $\bar{L}$ may be determined by using the following equation, obtained in [3]:

$$
\bar{L}=\frac{\sqrt{n}-1}{2 \operatorname{tg} \frac{\alpha}{2}}
$$

In [2], the authors provide information (in tabular form) about the values of $\zeta_{d}$, which after being approximated in [3], may be described by the following equation:

$$
\zeta_{d}=\left(0.000393 \alpha^{2}-0.00835 \alpha+0.091\right) \frac{n}{2} .
$$

Another option for estimating $\zeta_{d}$, given in [3], represents: 


$$
\zeta_{d}=2.6(1+0.8 \lambda)\left(1-\frac{1}{n}\right)^{2} \sin \frac{\alpha}{2} .
$$

In case of a system using an outlet diffuser the values concerning $\zeta_{d, \text { out }}$ are published in [2]. The data is given in tabular form and depending on the diffuser degree of expansion $n$ (area ratios), as well as the angle $\alpha$ and Reynolds number $R e$. Analyzing the provided information gives clear indications that the minor loss coefficient $\zeta$ is highly depended by the ratio $\frac{v_{\max , 2}}{v_{2}}$, related to the diffuser input section, where $v_{\max , 2}$ is the maximal velocity of the fluid flow in section 2-2 (fig.2), and $v_{2}=\frac{q}{\rho S_{2}}=\frac{Q}{S_{2}}=\frac{4 Q}{\pi d^{2}}$ is the average velocity concerning the same section. In this case, $q[\mathrm{~kg} / \mathrm{s}]$ and $Q\left[\mathrm{~m}^{3} / \mathrm{s}\right]$ are the mass and volumetric flow rates of the fluid flow, respectively, and $S_{2}=\frac{\pi d^{2}}{4}$ is the cross-section area of the diffuser input section.

The data concerning $\zeta_{d, \text { out }}$, published in [2], are valid in case that: $\frac{v_{\max , 2}}{v_{2}} \approx 1(l / d \approx 0)$ and $\frac{v_{\max , 2}}{v_{2}}>1(l / d \geq 10)$.

In [4], the authors present experimental data concerning $\zeta$, obtained at different angles, area ratios and wall roughness of the analyzed cone diffusers, that are also having different values of the relative length $l / d$ of the diffuser straight section: $l / d=1 ; 4 ; 6 ; 9$. It is clear from the data analysis that the value of $\zeta$ increases with the increasing of the ratio $l / d$ (and therefore $\frac{v_{\max , 2}}{v_{2}}$ ).

Fig.3 and table 1 present results concerning $\zeta_{d, \text { out }}$, obtained by using the equations (3), (4), (6) and (7).

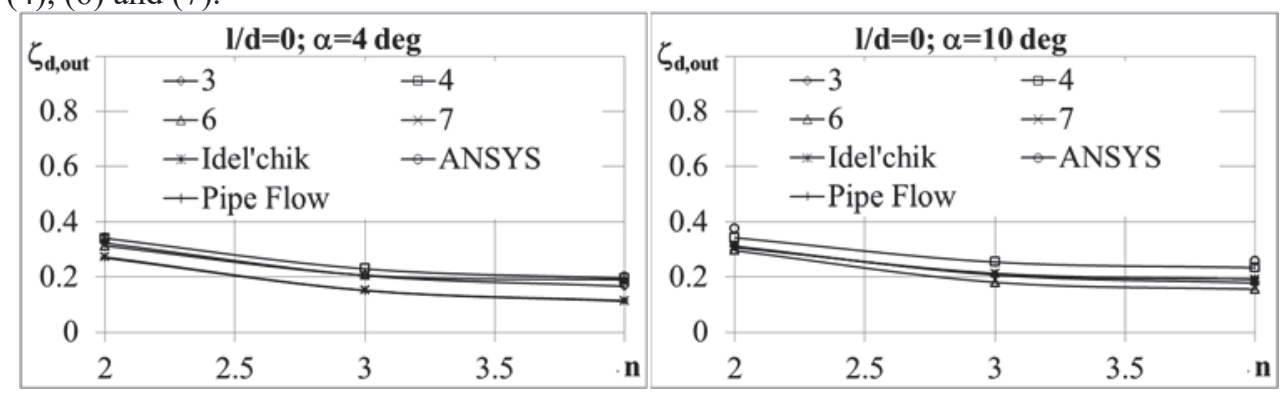

Fig. 3. Presenting the relationship $\zeta_{d, \text { out }}=f(n)$, obtained at different conditions.

In addition, data published in [2], denoted by "Idel'chik", are given for the case when $l / d=0$, as well as results obtained after modeling the flow in a diffuser by using ANSYS CFX. Values of $\zeta_{d, \text { out }}$, taken by the computational software product Pipe Flow Expert, used for accomplishing hydraulic estimation of pipe systems, are also given. Since the equations (3), (4), (6) and (7) are intended for determining the values of $\zeta_{d}$ in case of installing the 
diffuser between two pipes, the estimation of $\zeta_{d, \text { out }}$ may be accomplished by using the following equation [2]:

$$
\zeta_{d, o u t}=\zeta_{d}+\alpha_{\text {out }, d} \frac{1}{n^{2}}
$$

Indicating the lack of data (information) concerning the coefficient of Coriolis $\alpha_{\text {out }, d}$, it is assumed that $\alpha_{\text {out }, d}=1$. The major loss coefficient is considered to be: $\lambda=0.023$. The calculations are performed at the following conditions: area ratios $n=2,3,4$ and angles $\alpha=4 \mathrm{deg}$ and $\alpha=10 \mathrm{deg}$. According to [2], for these values of $n$ and $\alpha$ the diffusers are realized without detaching the fluid flow.

Table 1. The values of $\zeta_{d, \text { out }}$, obtained by using different relationships.

\begin{tabular}{|c|c|c|c|c|c|c|c|c|}
\hline$\alpha$ & $\mathbf{n}$ & \multicolumn{4}{|c|}{ Relationship № } & \multirow{2}{*}{ Idel'chik } & \multirow{2}{*}{$\begin{array}{c}\text { ANSYS } \\
\text { CFX }\end{array}$} & \multirow{2}{*}{$\begin{array}{l}\text { Pipe } \\
\text { Flow }\end{array}$} \\
\hline deg & - & 3 & 4 & 6 & 7 & & & \\
\hline 4 & 2 & 0.324 & 0.341 & 0.314 & 0.273 & 0.342 & 0.342 & 0.270 \\
\hline 4 & 3 & 0.206 & 0.230 & 0.207 & 0.152 & - & & 0.151 \\
\hline 4 & 4 & 0.167 & 0.196 & 0.190 & 0.114 & 0.186 & 0.201 & 0.113 \\
\hline 10 & 2 & 0.313 & 0.344 & 0.297 & 0.308 & 0.31 & 0.377 & 0.31 \\
\hline 10 & 3 & 0.208 & 0.255 & 0.181 & 0.214 & - & & 0.211 \\
\hline 10 & 4 & 0.179 & 0.235 & 0.156 & 0.192 & 0.182 & 0.260 & 0.193 \\
\hline
\end{tabular}

Analyzing the data, presented in fig. 3 and table 1, clearly indicates that the values of $\zeta_{d, \text { out }}$, obtained by using the software product Pipe Flow Expert, correspond to the results, obtained by using equation (7). The results, obtained by using equation (3), have similar values with the data, published in [2] after being approximated by using equation (6). There is a sufficient overlapping between the results obtained by using ANSYS CFX and equation (4) and the data provided by Idel'chik (at $\alpha=4 \mathrm{deg}$ ), published in [2].

\section{Determining the minor loss coefficient of cone diffusers at $\mathrm{l} / \mathrm{d}<10$}

Sometimes, in practice, it is necessary to install a short pipe $(l / d<10)$ after the fan outlet. However, a review of the studied literature sources indicates the lack of information concerning the existing of analytical equations that may be used to determine $\zeta_{d \text {,out }}$ in such cases. This leads to difficulties in performing an analytical research for determining the impact of the diffuser on the fan system energy efficiency. Furthermore, it is hard to evaluate the impact of the flow velocity profile at the fan outlet on the diffuser minor loss coefficient in case that it is installed at the end of a booster pipe, as well as the impact of the distance between the fan and diffuser outlets on the value of the same coefficient. Such relationships may be determined by CFD modeling of the fluid flows, using modern computational software products.

In the recent years it is widely used to modeling different processes, related to heat and mass transfer and fluid motion, by using CFD software products [5-8]. Validating of such models by using experimental data enables the accomplishment of planned numerical experiments. This leads to the achieving of significant saving of time and money, required in performing real experiments. In this current work it has been established CFD models of cone diffusers, using different combinations of parameters (diffuser angle $\alpha$, area ratio $n$ and relative length $l / d$ ). 
The models are validated by using experimental data, published in [4], and may be used for numerical modeling and analytical analyzing of the energy efficiency of fan systems.

For accomplishing the numerical calculations it has been used ANSYS CFX. The generating of the calculation meshes is performed by using the universal generator ICEM CFD. The analyzed diffusers are studied at the following air properties: temperature $25^{\circ} \mathrm{C}$, density $\rho=1.185 \mathrm{~kg} / \mathrm{m}^{3}$ and dynamic viscosity $\mu=0.00001831 \mathrm{~Pa} \cdot \mathrm{s}$. For performing the computational procedures the turbulent model SST (Shear Stress Transport) is selected. It represents a hybrid model, containing a combination of $k-\varepsilon$ and $k-\omega$, which in case flows having flow separation is considered to be the most proper option for modeling. Such flows are typical for diffusers with a concrete value of their angle $\alpha$. In [9-11], the authors provide detailed information concerning the equations describing the previously mentioned model. For determining accurately the necessary and sufficient density of the computational mesh it has been studied four types of meshes having the following parameters: area ratio $n=\frac{D^{2}}{d^{2}}=2$ and diffuser angle $\alpha=16 \mathrm{deg}$. In front of the diffuser there is a straight pipe section with a relative length $l / d=4$. The diameter of the straight pipe section, which is equal to the diffuser inlet diameter, is set to be $d=50 \mathrm{~mm}$. The Reynolds number, estimated with the relevant parameters related to the diffuser inlet, respectively is $R e=\frac{v_{2} d \rho}{\mu}=200000$. To ensure this current value of $R e$ at the entrance of the rectilinear section (1-1 in fig.1), a uniform, normal cross-sectional speed $v_{1}=61.806 \mathrm{~m} / \mathrm{s}$, is set. The diffuser minor loss coefficient, in case that it is installed at the pipe system outlet, includes the kinetic energy loss $\rho \frac{v_{3}^{2}}{2}$. According to the scheme, given in fig.2, the value of $\zeta$ is estimated by using the following equation [12]:

$$
\zeta=1-\frac{2\left(p_{3}-p_{2}\right)}{\rho v_{2}^{2}},
$$

where $p_{2}$ and $p_{3}$ is the static pressure in sections 2-2 and 3-3, respectively.

The number of elements and values of $\zeta$, obtained after accomplishing a numerical simulating of the four analyzed meshes, are given in table 1 .

Table 2. Characteristics of the analyzed computational meshes.

\begin{tabular}{|c|c|c|}
\hline Name & $\begin{array}{c}\text { Number of } \\
\text { elements, } \mathbf{N}\end{array}$ & $\boldsymbol{\zeta}$ \\
\hline Mesh 1 & 193104 & 0.510002528 \\
\hline Mesh 2 & 379989 & 0.50612875 \\
\hline Mesh 3 & 530009 & 0.511288234 \\
\hline Mesh 4 & 8964399 & 0.517850515 \\
\hline
\end{tabular}

In fig.4, it is graphically presented the following relation: $\zeta_{d, \text { out }}=f(N)$. From the data, given in table 2 and fig. 4 , it may be seen that even if the number of the computations is increased almost 17 times - from 530009 to 8 964399, the change of $\zeta_{d, \text { out }}$ is insignificant 
$-1,27 \%$. For this reason, and in order to save computational resources, Mesh 3 is selected to perform the simulation calculations. It is considered for it to be an unstructured mesh, having a core containing 85709 tetrahedras with size of global max element $0.0031 \mathrm{~m}$. There is a prism layer at the walls of the straight section in front of the diffuser, as well as on the diffuser, containing 30 layers and its height ratio is 1.21. The prism layer has a height of $1.10^{-5} \mathrm{~m}$, and the total prism layer height is considered to be $0.0144515 \mathrm{~m}$. The number of the prismatic elements is set to be 444300 .

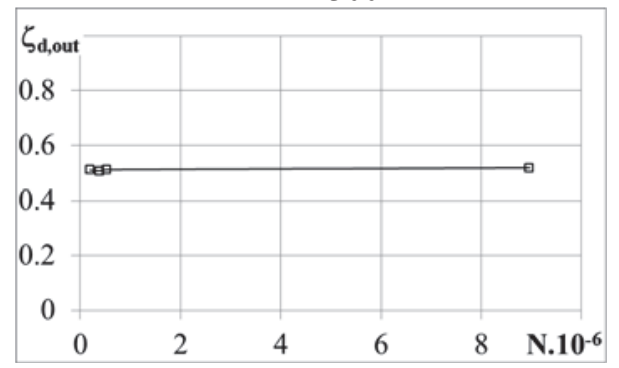

Fig. 4. A relationship concerning $\zeta_{d, \text { out }}$, obtained after numerical modeling of the number of cells of the computing mesh is accomplished.

For the aims of this research work 24 models with the parameters of Mesh 3, having different combinations of a diffuser angle $(\alpha=4,6,8$ and $10 \mathrm{deg})$, area ratio $(n=2,3,4$ ) and relative length $(l / d=6$ and 9$)$ of the straight pipe section in front of the diffuser, are generated.

\section{Results and discussion}

Fig. 5,a provides information concerning the velocity distribution of a diffuser having the following parameters: $n=2, \alpha=10 \mathrm{deg}$ and $l / d=6$, that is obtained as a result of accomplishing numerical simulations. The velocity distribution in section 2-2 is given in fig.5,b. The average velocity of this section and equation (9) are used to estimate $\zeta_{d, \text { out }}$.

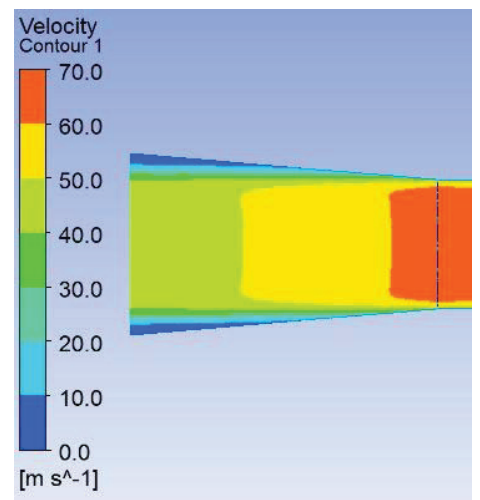

a).

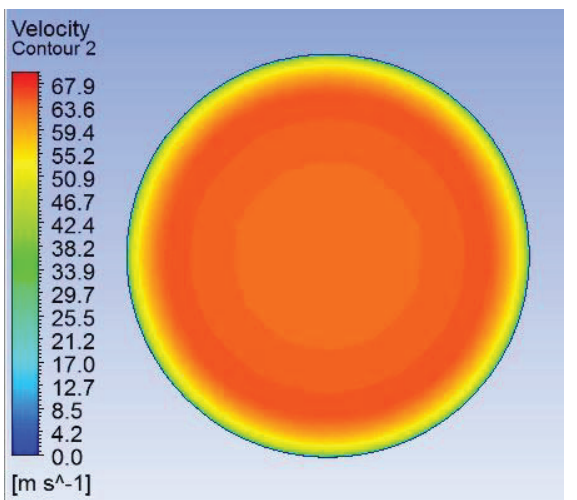

b).

Fig. 5. Velocity distribution for a model with $n=2, \alpha=10 \mathrm{deg}$ and $l / d=6$ : a). in the diffuser; b). in section 2-2.

The results, obtained after performing the numerical simulations of the designed models, are given in table 3 and some of them are graphically presented in fig.6. The columns of table 3 , denoted with 1 , contain experimental data, published in [4], while the ones denoted with 2 contain results, obtained after numerical simulations are performed. 
The graphical results are presented in similar (analogical) way - curve 1 is related to the experimental data, while curve 2 concerns the numerical simulation results. The experimental data, published in [4], is graphically presented. In order to use these graphs they have to be preliminary digitalized by using a free software product.

Table 3. Results, obtained after performing the computational simulations.

\begin{tabular}{|c|c|c|c|c|c|}
\hline$\alpha$ & $\mathbf{n}$ & \multicolumn{2}{|c|}{$\mathbf{l} \mathbf{d = 6}$} & \multicolumn{2}{c|}{$\mathbf{l} \mathbf{d = 9}$} \\
\hline $\mathrm{deg}$ & - & 1 & 2 & 1 & 2 \\
\hline 4 & 2 & 0.371 & 0.376 & 0.422 & 0.395 \\
\hline 4 & 3 & 0.257 & 0.268 & 0.306 & 0.288 \\
\hline 4 & 4 & 0.207 & 0.228 & 0.255 & 0.243 \\
\hline 6 & 2 & - & 0.406 & - & 0.434 \\
\hline 6 & 3 & - & 0.31 & - & 0.334 \\
\hline 6 & 4 & - & 0.263 & - & 0.286 \\
\hline 8 & 2 & - & 0.446 & - & 0.478 \\
\hline 8 & 3 & - & 0.351 & - & 0.375 \\
\hline 8 & 4 & - & 0.212 & - & 0.317 \\
\hline 10 & 2 & 0.461 & 0.484 & 0.511 & 0.511 \\
\hline 10 & 3 & 0.368 & 0.368 & 0.421 & 0.410 \\
\hline 10 & 4 & 0.321 & 0.329 & 0.375 & 0.351 \\
\hline
\end{tabular}
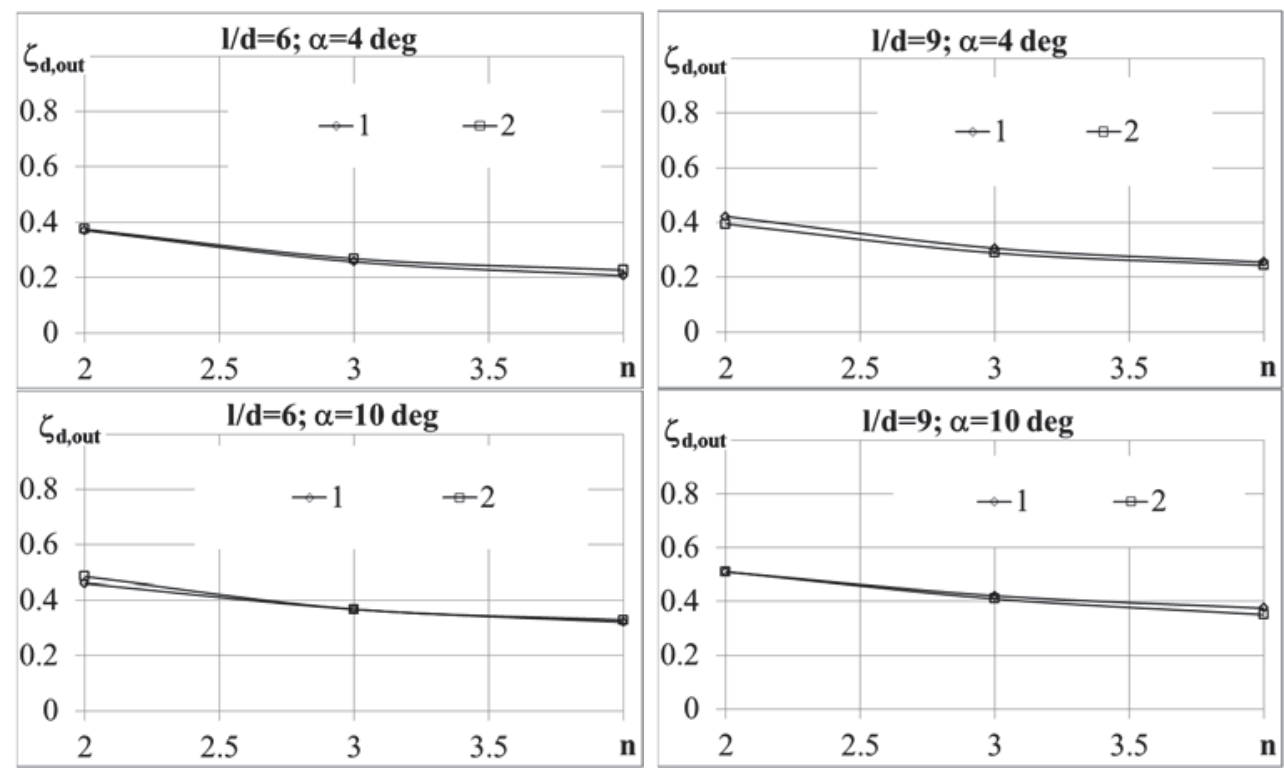

Fig. 6. Graphical relationships $\zeta_{d, \text { out }}=f(n)$, obtained at applying different combinations of $\alpha$ and $l / d: 1$ - experimental data [4]; 2 - numerical simulations.

To evaluate the results, obtained after performing numerical simulations, the relative error (inaccuracy) $\Delta$, given in percentage, of each model has to be determined. It may be estimated as it follows:

$$
\Delta=\frac{\left|\zeta_{1}-\zeta_{2}\right|}{\zeta_{1}} \cdot 100 \%
$$


wher $\zeta_{1}$ is determined after performing experiments, and $\zeta_{2}$ - obtained after performing numerical simulations. The results are given in table 4 .

Table 4. Relative error of the results, obtained after performing numerical modelling.

\begin{tabular}{|c|c|c|c|}
\hline$\alpha$ & $\mathbf{n}$ & $\mathbf{1} / \mathbf{d}=\mathbf{6}$ & $\mathbf{1} / \mathbf{d}=\mathbf{9}$ \\
\hline $\mathrm{deg}$ & - & & 6.5273175 \\
\hline 4 & 2 & 1.1265512 & 5.6370539 \\
\hline 4 & 3 & 4.3957351 & 4.5630719 \\
\hline 4 & 4 & 9.8358713 & 0.0108422 \\
\hline 10 & 2 & 5.1198163 & 2.7847239 \\
\hline 10 & 3 & 0.0860164 & 6.3870104 \\
\hline 10 & 4 & 2.5214154 & \multicolumn{2}{|c}{} \\
\hline
\end{tabular}

From the data, given in table 4 , it may be seen that the models provides acceptable results, in terms of their accuracy, for engineering calculations. The data from the columns 2 in table 3 , have been preliminary approximated by using the following equation:

$$
\zeta_{d, \text { out }}=\left(a \cdot \alpha^{2}+b \cdot \alpha+c\right) n^{d},
$$

where $\alpha$ is given in degrees. Table 5 provides information concerning the values of the coefficients $a, b, c$ and $d$.

Table 5. Values of the coefficients $a, b, c$ and $d$.

\begin{tabular}{|c|c|c|c|c|c|}
\hline & $\boldsymbol{a}$ & $\boldsymbol{b}$ & $\boldsymbol{c}$ & $\boldsymbol{d}$ & $\begin{array}{c}\text { Coefficient of } \\
\text { determination } \boldsymbol{R}^{2}\end{array}$ \\
\hline$l / d=6$ & 0.00208 & 0.003654 & 0.5658 & -0.7156 & 0.9351 \\
\hline$l / d=9$ & -0.0009522 & 0.04836 & 0.4005 & -0.6024 & 0.9923 \\
\hline
\end{tabular}

This equation, proposed by the authors, may be used in performing analytical studying concerning the energy efficiency of fan systems, having installed outlet diffusers.

\section{Conclusion}

This paper presents the results of studying various physical relationships, used for determining the minor loss coefficient of a cone diffuser, in case that it is installed at the system outlet. The results are compared with experimental data and numerical results, obtained after using ANSYS CFX and the data base of Pipe Flow Expert. It is found that the results of the studied relationships are valid (applicable) in case of a uniform velocity profile of the low at the diffuser inlet or in case of a lack of straight pipe section in front of it.

For determining the minor loss coefficient of a cone diffuser, being installed at the system outlet and having a straight pipe section in front of it, some models in ANSYS CFX are established. These models are validated by using previously published experimental data. The results obtained are being preliminary approximated by using the equation $\zeta_{d, \text { out }}=f(n ; \alpha)$, enabling the possibility for performing analytical studies concerning the energy efficiency of fan systems, having outlet diffusers.

This paper contains results found after working on a project 2020-FAI-02, funded by the Fund "Scientific research" of the University "Angel Kanchev" of Ruse, Bulgaria. 


\section{References}

1. A. Bruk, T. Matikashvili, M. Nevelson, G. Raer, T. Solomahova, E. Yudin, Centrobezhnaye ventilyataray. Mashinostroenie, (Moskva, 1975)

2. I. Idel'chik. Spravochnik po gidravlicheskim soprotivleniyam. Gosudarstvennoe Energeticheskoe Izdatel'stvo (Moskva-Leningrad, 1960)

3. Y. Gotseev, A. Obukhovskiy, S. Salenko. On head losses in conical diffusers. AIP Conference Proceedings 2027, 030048 (2018).

https://aip.scitation.org/doi/abs/10.1063/1.5065142

4. L. Voytovich, G. Emel'yanova. Experimentol'noe I teoreticheskoe issledovanie gidravlicheskovo soprovleniya konicheskih difuzorov s sherohovatoy poverhnostyu. Uchenaye zapiski CAGI, XVI, 4 (1985)

5. Z. Kolev, S. Kadirova. CFD simulation of forced heat transfer of gas in pipe. E3S Web of Conferences 112, 01008 (2019), TE-RE-RD 2019, E-ISSN:2267-1242

6. Z. Kolev, S. Kadirova. Numerical Modelling of Heat Transfer in Convector's Pipes by ABAQUS. IOP Conf. Series: Materials Science and Engineering 595 (2019) 012006, NACOT 2019

7. K. Krumov, N. Penkova, Numerical analysis of the transient heat transfer in high temperature chamber furnaces, IOP Conference Series: Materials Science and Engineering, Volume 595 (1), 2019, 012005

8. N. Penkova, P. Chervenliev, B.. Mladenov, K. Krumov, Modelling and numerical simulation of mass transfer and mechanical processes at ceramic ware in industrial dryers, Proceedings of 6th European Conference on Computational Mechanics and the 7th European Conference on Computational Fluid Dynamics; ISBN: 978-84-947311-67; 2018, 4368-4379, http://www.eccm-ecfd2018.org/frontal/docs/Ebook-Glasgow2018-ECCM-VI-ECFD-VII.pdf

9. ANSYS, Inc, ANSYS CFX-Solver Theory Guide, November 2011.

10. I. Antonov, R. Velichkova, S. Antonov, Differential Equations for Turbulent Flow, PROCEEDINGS of the SCIENTIFIC STUDENT SESSION - SSS'14, 53 (2014) http://conf.uni-ruse.bg/bg/docs/sns/2014/AIF-1.pdf

11. A. Terziev, Specifics in numerical modeling of flow past a square-cylinder, PROCEEDINGS of the SCIENTIFIC STUDENT SESSION - SSS'14, 53 (2014) http://conf.uni-ruse.bg/bg/docs/sns/2014/AIF-1.pdf

12. D. Miller, Internal flow systems, BHRA, The Fluid Engineering Centre (1990) 